\title{
The latest developments on Of?p stars
}

\author{
Yael Nazé ${ }^{1}$, Asif ud-Doula ${ }^{2}$, Maxime Spano ${ }^{3}$, Gregor Rauw ${ }^{1}$, Michael \\ De Becker ${ }^{1}$ and Nolan R. Walborn ${ }^{4}$ \\ ${ }^{1}$ FNRS-ULg, Dept AGO, Allée du 6 Août 17, Bât B5C, B4000-Liège, Belgium, \\ email: naze@astro.ulg.ac.be \\ ${ }^{2}$ Penn State Worthington Scranton, USA; ${ }^{3}$ Obs. Genève, Switzerland; ${ }^{4}$ STScI, USA
}

\begin{abstract}
In recent years several in-depth investigations of the three prototypical Of?p stars were undertaken, revealing their peculiar properties. To clarify some of the remaining questions, we have continued our monitoring of the prototypical Of?p trio. HD 108 has now reached its quiescent, minimum-emission state, for the first time in 50-60yrs, while new echelle spectra of HD 148937 confirm the presence in several $\mathrm{H}$ and He lines of the $7 \mathrm{~d}$ variations detected previously only in the $\mathrm{H} \alpha$ line. A new XMM observation of HD 191612 clearly shows that its Xray emission is not modulated by the orbital period of $1542 \mathrm{~d}$, but the high-energy variations are rather compatible with the 538d period of the optical changes - it is thus not of colliding-wind origin but linked to the phenomena responsible for the spectral/photometric variations, though our current MHD simulations remain at odds with the observational properties.
\end{abstract}

Keywords. X-rays: stars, stars: early-type, stars: individual (HD 108, HD 148937, HD 191612), stars: emission-line

\section{Introduction}

The Of?p category was proposed 40 years ago to gather a few O-type stars presenting peculiarities in their optical/UV spectrum. These objects were then known to display strong C III 4650 emission lines but recent studies showed that this is not their sole peculiar characteristics (in fact, at the present time, stars displaying "only" strong C III 4650 emission should be classified as Ofc, see Walborn et al. 2010). Of?p stars also possess: narrow $\mathrm{P}$ Cygni/emission features in Balmer, HeI,... lines which vary in a recurrent manner; correlated photometric variations; strong magnetic fields; and large X-ray overluminosities (for a review, see Nazé et al. 2008a). Five Galactic Of?p stars are known, but only the first three stars which were identified as such in the 1970s (the so-called "prototypical" objects HD 108, HD 148937, HD 191612) have been extensively studied, though some questions remained open about their physical properties. We have obtained additional data with the aim to contribute to the understanding of these peculiar objects (see Nazé et al. 2010).

\section{HD 108}

HD 108 is the first Of?p for which long-term spectral variations were reported (Andrillat et al. 1973, Nazé et al. 2001). However, the recurrence timescale appeared very long, about 55 yrs (Nazé et al. 2006). This value was not well constrained because of the lack of detailed observations over that period (often, only the line shape - emission/absorption/P Cygni - is mentioned). The Liège team has been closely monitoring HD 108 from 1986 on at the Observatoire de Haute-Provence. Since the beginning, the data showed a continuous decline in the emission strengths. This decrease has now stopped, as the spectra taken between 2005 and 2009 appear similar: the star has thus 
reached its quiescence state for the first time since the 1950s. It remains to be seen when a new cycle will begin. Indeed, the knowledge of the quiescence time will be very useful to better constrain the systems geometry.

\section{HD 148937}

A previous 3-yrs spectral monitoring revealed small-amplitude (a few percent of the peak's amplitude) variations of the $\mathrm{H} \alpha$ line, with a potential period of $7 \mathrm{~d}$ (Nazé et al. 2008b). While the X-ray data were undistinguishable from those of HD 108 and HD 191612, the optical properties thus appeared quite different from those of the other two "prototypical" Of?p stars (which display large variations of many lines, occurring with a long period and correlated with rather large photometric changes). However, the quality of this first monitoring was quite limited: the data sampling (monthly exposures) and spectral resolution $(\mathrm{R} \sim 2300)$ were not really fit to detect subtle variations occurring on short timescales. A new short-term monitoring with daily exposures taken with higher resolution ( $\mathrm{R} \sim 55000)$ was performed at the La Silla Observatory. Variations in some He I and He II 4686 lines were detected (but not in C III 4650) and the 7d period was confirmed for all ! varying lines. HD 148937 is thus similar to the other two "prototypical" Of?p stars, though with a shorter period: the small-amplitude is most probably related to a geometrical effect (e.g. a low inclination of the star's rotation axis in the scenario of an oblique rotator with magnetically-confined winds).

\section{HD 191612}

A previous X-ray monitoring (Nazé et al. 2007) unveiled some variations of the highenergy emission, but their cause was not ascertained: they could either be related to the binary nature of the star $\left(P_{\text {orb }}=1542 d\right)$ through colliding-wind emission or to the line profile variations $\left(P_{c y c}=538 d\right)$ typical of the Of?p phenomenon. Note that the old data were taken at orbital phases $\phi_{\text {orb }}=0.83-0.96$ and at phases $\phi_{c y c}=0.09-0.44$ in the line profile cycle. A new XMM dataset was taken at a different orbital phase $\left(\phi_{\text {orb }}=0.55\right)$ but at a similar phase in the spectral cycle $\left(\phi_{c y c}=0.13\right)$. After analysis, the recorded emission appears very similar to the previous data: a colliding-wind origin can thus be rejected.

To explain the variations and the X-ray overluminosity, a scenario involving magnetically-confined winds and oblique rotation was proposed. This can be modelled using MHD simulations, taking into account the known stellar properties. The predicted situation is a bright $\mathrm{X}$-ray emission from a very hot plasma $(>10 \mathrm{MK})$, produced close to the star (hence X-ray lines should be narrow). While the overluminosity is indeed observed, the data rather favor a cooler plasma and quite broad X-ray lines. Work still remains to be done until the X-ray emission of the Of?p stars will be fully understood.

\section{References}

Andrillat, Y., Fehrenbach, C., Swings, P., \& Vreux, J. M. 1973, A\&A, 29, 171

Nazé, Y., Vreux, J.-M., \& Rauw, G. 2001, A\&AA, 372, 195

Nazé, Y., Barbieri, C., Segafredo, A., Rauw, G. et al. 2006, IBVS 5693

Nazé, Y., Rauw, G., Pollock, A. M. T., Walborn, N. R. et al. 2007, MNRAS, 375, 145

Nazé, Y., Walborn, N. R., \& Martins, F. 2008a, Rev. Mexicana AyA 44, 331

Nazé, Y., Walborn, N. R., Rauw, G., Martins, F. et al. 2008b, AJ, 135, 1946

Nazé, Y., Ud-Doula, A., Spano, M., Rauw, G. et al. 2010, A $\& A$ 520A, 59

Walborn, N. R., Sota, A., Maíz Apellániz, J., Alfaro, E. J. et al. 2010, ApJ (Letters), 711, L143 